\title{
Periorquitis meconial. A propósito de un caso
} Meconium periorchitis. A case report

\author{
Dra. Pamela Acosta ${ }^{a}$, Dr. Francisco Gambina ${ }^{a}$, Dr. Lucas Perelli ${ }^{a}$, Dr. Estanislao Díaz Pumaráa, \\ Dr. Jorge L. Martínez $z^{b}$ Dra. María N. Etchepareborda y Dr. Luis Zuccardib
}

\section{RESUMEN}

La periorquitis meconial es infrecuente. En el feto, está abierto el conducto peritoneo vaginaly, ante una perforación intestinal por cualquier causa (atresia intestinal, vólvulo, entre otras), se produce una peritonitis meconial. El contenido intestinal puede pasar hacia la cavidad vaginal escrotal. La peritonitis meconial puede resolverse espontáneamente y sin consecuencias. Restos calcificados de este evento pueden quedar en la cavidad peritoneal y/o en el escroto. En el recién nacido, se observará unhidrocele y una masa palpable intraescrotal, ecográficamente heterogénea y con calcificaciones. La periorquitis meconial o vaginalitis meconial se resuelve espontáneamente. Su desconocimiento podría llevar a cirugías innecesarias en el neonato.

Se presenta el caso de un paciente de 33 días de vida con una masa escrotal, en quien se realizó una cirugía exploratoria. El diagnóstico anatomopatológico fue de periorquitis meconial. Palabras clave: periorquitis meconial, vaginalitis meconial, masa paratesticular, masa escrotal, neonato.

\begin{abstract}
Meconium periorchitis is uncommon. In the unborn child the peritoneum vaginal canal is open and, secondary to intestinal perforation due to any cause (intestinal atresia, volvulus, and others), meconium peritonitis occurs. The intestinal content reaches the scrotal vaginal cavity. Meconium peritonitis can heal spontaneously and without consequences. Calcified remnants of this event may remain in the peritoneal cavity and / or scrotum. In the newborn, a hydrocele and scrotal mass can be observed; the ultrasound will show a heterogeneous image with calcifications. Meconium periorchitis or meconium vaginalitis resolves spontaneously. The lack of awareness of this disease could lead to unnecessary surgery in the newborn. We present a 33 days old patient with a scrotal mass in whom surgery was performed with the pathological diagnosis of meconium periorchitis.

Key words: meconium periorchitis, meconium vaginalitis, paratesticular mass, scrotal mass, neonate.
\end{abstract}

http:/ / dx.doi.org/10.5546/aap.2015.e330

a. Clínica Pediátrica.

b. Cirugía Pediátrica.

Hospital de Niños Ricardo Gutiérrez,

Ciudad Autónoma de Buenos Aires.

Correspondencia:

Dra. Pamela Acosta: pameacosta@hotmail.com

Financiamiento: Ninguno.

Conflicto de intereses: Ninguno que declarar.

Recibido: 3-3-2015

Aceptado: 11-6-2015

\section{INTRODUCCIÓN}

La periorquitis meconial es una causa poco frecuente de masa escrotal benigna, producto de una perforación intestinal intrauterina, lo que genera un cuadro de peritonitis meconial. ${ }^{1}$ A través del proceso vaginal permeable, puede llegar al escroto y ocasionar una masa paratesticular en el neonato., ${ }^{2,3}$

Debido a su baja prevalencia, no suele considerarse dentro de los diagnósticos probables, lo que lleva a cirugías, muchas veces innecesarias. ${ }^{4} \mathrm{Su}$ diagnóstico es clínico, apoyado en el diagnóstico por imágenes, esencialmente en la ecografía de partes blandas, que permite identificar los testículos y los procesos paratesticulares.

\section{PRESENTACIÓN DEL CASO}

Se trata de un paciente de 33 días de vida, derivado por su pediatra de cabecera al consultorio de cirugía por notar en la consulta una tumoración escrotal izquierda duro elástica que parecía indolora.

Es un niño nacido de término (40 semanas), con alto peso para la edad gestacional (4750 g), sin antecedentes perinatológicos de relevancia. Hijo de madre primigesta de 19 años, con un embarazo adecuadamente controlado, serologías para VIH, hepatitis B, Chagas, VDRL y toxoplasmosis del tercer trimestre negativas, sin complicaciones. No refiere uso de fármacos durante la gestación.

Al momento del examen físico, se encuentra en buen estado general, con adecuado progreso pondoestatural y presenta como datos positivos hidrocele izquierdo, asociado a una tumoración escrotal homolateral de aproximadamente $2 \mathrm{~cm}$ de diámetro, de consistencia duro elástica, sin signos de flogosis local, con ambos testículos palpables en bolsas escrotales.

Se realizan hemograma, función renal, hepatograma y dosaje de alfafetoproteína y subunidad beta de gonadotrofina coriónica humana, todo dentro de los límites normales para la edad. Se solicita una ecografía testicular, que muestra una formación heterogénea, con calcificaciones de contornos lobulados de 29 × 17 
x $20 \mathrm{~mm}$, contigua al margen medial y proximal del testículo izquierdo (Figura 1).

Ante la presencia de masa escrotal de características duro elásticas, adherida a la pared escrotal, sin la certeza de haber estado presente desde el nacimiento, se sospecha una patología oncológica testicular, por lo que se toma una conducta quirúrgica, por vía inguinal, sin solicitud de biopsia por congelación. En el acto quirúrgico, se constatan ambos testículos indemnes y se evidencia la persistencia de la tumoración adherida a la pared escrotal (Figura 2), por lo que se decide conservar ambos testículos.

Se procede a la exéresis de dicha tumoración y se envía el material para el estudio anatomopatológico. Finalmente, se realiza orquidopexia izquierda.

Presenta buena evolución en el posoperatorio inmediato, y se otorga el egreso hospitalario a las $24 \mathrm{~h}$.

Se recibe, posteriormente, el resultado de anatomía patológica, que informa masa irregular de característica fibrosa, congestiva, con numerosos focos de calcificación, macrófagos cargados con pigmento, vasocongestión, hemorragia y edema de partes blandas, compatible con periorquitis meconial.

FIGURA 1. Ecografía testicular
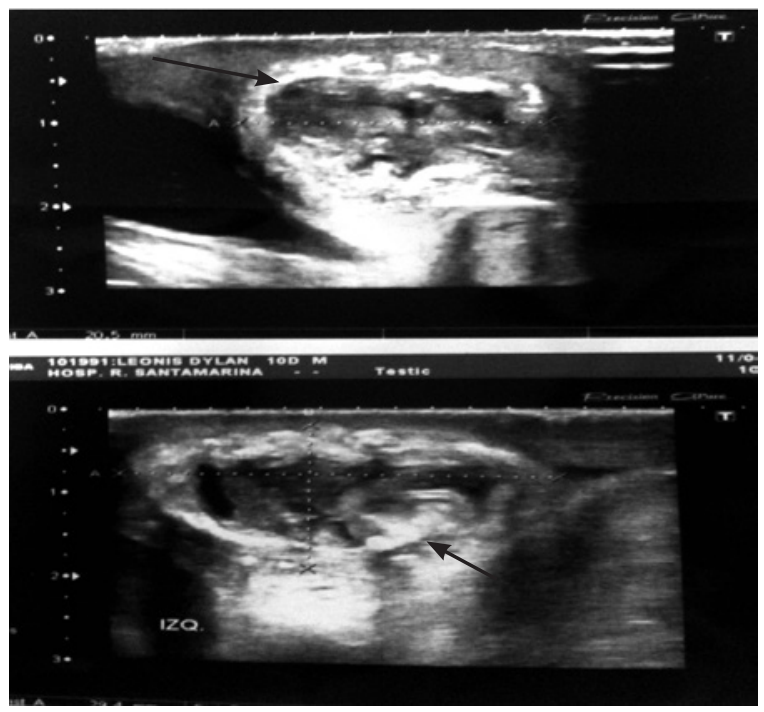

Se evidencia una formación heterogénea con calcificaciones de contornos lobulados de $29 \times 17$ × $20 \mathrm{~mm}$, contigua al margen medial y proximal del testículo izquierdo.

\section{DISCUSIÓN}

La periorquitis meconial es una entidad poco frecuente, cuya incidencia no está determinada. La bibliografía sobre esta patología consiste en reportes de casos o serie de casos. Como en el paciente presentado, se manifiesta habitualmente como una masa escrotal entre el nacimiento y los 20 meses de vida, con una media, al momento del diagnóstico, de un mes. ${ }^{1}$

El proceso se inicia cuando existe una perforación intestinal intraútero, que permite el pasaje de meconio al peritoneo y produce un cuadro de peritonitis química, llamado peritonitis meconial, que, si bien suele ser un cuadro grave de alta mortalidad, puede autolimitarse. Cuando este proceso se extiende al escroto a través del conducto peritoneo vaginal permeable, se denomina periorquitis meconial. Los factores de riesgo para la perforación incluyen alteraciones intestinales, como vólvulo, atresia o compromiso circulatorio, ${ }^{1-3}$ y el uso de antiinflamatorios no esteroides por parte de la madre. ${ }^{3}$ En el paciente presentado, no surgen antecedentes obstétricos que justifiquen este cuadro.

La periorquitis meconial se manifiesta inicialmente como hidrocele o tumoración escrotal evidente al nacer. ${ }^{5}$ No suelen observarse signos de flogosis. Durante la evolución, el meconio dentro del escroto induce una respuesta inflamatoria local que determina un aumento de volumen de los tejidos adyacentes y un

\section{FIgURA 2. Abordaje quirúrgico por vía inguinal}

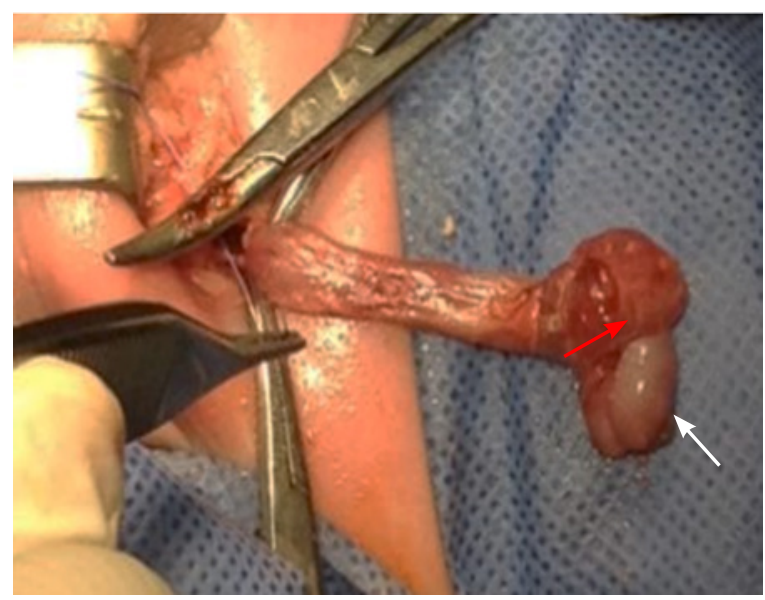

Se observa una tumoración adherida a la pared escrotal (flecha roja) con testículo indemne (flecha blanca). 
aumento de consistencia debido al depósito de calcio. Con la palpación, habitualmente, es posible separar la masa del testículo. El examen físico completo orientará la etiología. Dentro de los diagnósticos diferenciales, se deberán considerar tumores de células germinales, como seminoma, teratomas o gonadoblastoma, tumores gonadales no germinales, torsión testicular, hernias inguinoescrotales y hematomas. ${ }^{2}$

Como exámenes complementarios, el estudio de elección para el abordaje de esta patología es la ecografía, ${ }^{7}$ ya que, además de su accesibilidad e inocuidad, permite diferenciar entre procesos testiculares y paratesticulares. Su limitación radica en que es un estudio dependiente del operador. En la mayoría de los casos, se evidencia una imagen hiperecogénica con sombra acústica posterior debido a las calcificaciones ${ }^{6}$ y ausencia de flujo en el estudio con doppler. ${ }^{2}$ Con el uso de este método, podría ser diagnosticada, incluso, de forma prenatal. La radiografía abdominal, si bien es poco sensible, es importante, ya que permite observar las calcificaciones peritoneales, aunque pueden estar ausentes hasta en el 10\% de los casos, lo que dificulta el diagnóstico. ${ }^{1,4}$ Varios autores sostienen que la presencia concomitante de calcificaciones abdominales y testiculares en un paciente con masa escrotal es diagnóstica de la entidad. ${ }^{5,7}$
Esta entidad es autolimitada, aunque la exploración quirúrgica estaría indicada en aquellos casos atípicos o en los cuales se plantearan dudas diagnósticas, como en el paciente presentado. ${ }^{5,7}$

\section{CONCLUSIÓN}

Si bien la periorquitis meconial es una entidad infrecuente, debería ser incorporada en el diagnóstico diferencial de las masas escrotales en los primeros meses de vida para, de esta manera, evitar intervenciones innecesarias.

\section{REFERENCIAS}

1. Herman TE, Siegel MJ. Meconium Periorchitis. J Perinatol 2004;24(1):53-5.

2. Alanbuki, AH, Bandi A, Blackford N. Meconium periorchitis: a case report and literature review. Can Urol Assoc J 2013;7(7-8):e495-8.

3. AliYA,LutfiSA. Acase of Meconium Periorchitis presenting as a scrotal mass. J Neonatal Perinatal Med 2009;2(1):61-6.

4. Garat JM, Alagaba F, Parra L, Gómez L. Meconium Vaginalitis. Br J Urol 1991;68(4):430-1.

5. Brown-Harrison MC, Harrison AM, Reid BS, Cartwright PC. Meconium Periorchitis - A cause of scrotal mass in the newborn. Clin Pediatr (Phila) 2000;39(3):179-82.

6. Damaledo P, Himawan S. Meconium periorchitis: An unusual cause of newborn scrotal mass. Med J Indones 2009;18(4):290-3.

7. Williams HJ, Abernethy LJ, Losty P, Kotiloglu E. Mechonium Periorchitis - a rare cause of a paratesticular mass. Pediatr Radiol 2004;34(5):421-3. 